\title{
Use of Mycelium and Detached Leaves in Bioassays for Assessing Resistance to Boxwood Blight
}

Yonghong Guo and Richard T. Olsen, U.S. Department of Agriculture/Agricultural Research Service, U.S. National Arboretum - Floral and Nursery Plants Research Unit, Beltsville, MD 20705; Matthew Kramer, U.S. Department of Agriculture/Agricultural Research Service, Statistics Group, Beltsville, MD 20705; and Margaret Pooler, U.S. Department of Agriculture/Agricultural Research Service, U.S. National Arboretum - Floral and Nursery Plants Research Unit, Beltsville, MD 20705

\begin{abstract}
Guo, Y. H., Olsen, R. T., Kramer, M., and Pooler, M. 2016. Use of mycelium and detached leaves in bioassays for assessing resistance to boxwood blight. Plant Dis. 100:1622-1626.

Boxwood blight caused by Calonectria pseudonaviculata is a newly emergent disease of boxwood (Buxus spp. L.) in the United States that causes leaf drop, stem lesions, and plant death. A rapid and reliable laboratory assay that enables screening hundreds of boxwood genotypes for resistance to boxwood blight is needed to enable breeding and selection of resistant cultivars. Using eight boxwood cultivars with differing susceptibilities, we examined parameters for a screening assay comparing whole plant inoculation with detached leaf inoculation, use of mycelium versus spores as the inoculum, comparison of times of the year for inoculation, and comparison of two leaf

inoculation methods. Inoculation of detached leaves gave comparable results to inoculation of whole plants when compared across genotypes, although the detached leaf assay resulted in greater percentages of symptom expression. The time of year of plant inoculation (spring, summer, or winter) did not affect the relative expression of symptoms among the most resistant and susceptible genotypes. Inoculating plants with mycelium was as effective as spore inoculation for causing disease symptoms and allowed us to distinguish the more resistant genotypes, yet mycelium inoculation was much easier to prepare in large quantities for multiple assays.
\end{abstract}

Boxwood blight caused by Calonectria pseudonaviculata Henricot (syn. Cylindrocladium pseudonaviculata, C. buxicola), is a newly emergent disease of boxwood (Buxus spp. L.) in the United States. The disease, which causes leaf drop, stem lesions, and plant death, is a significant threat to the boxwood industry, which has an annual market value of over $\$ 100$ million (USDA-NASS 2010). First identified in the United Kingdom in 1994 (Henricot et al. 2000), the disease has spread throughout continental Europe, parts of western Asia, and into North America (Elmhirst and Auxier 2013; Henricot and Culham 2002; Ivors et al. 2012; Malapi-Wight et al. 2014). In the United States, the first confirmed reports of the disease were made in Connecticut and North Carolina in November 2011 (Ivors et al. 2012), followed by diagnosis in 12 additional states and three Canadian provinces (Elmhirst and Auxier 2013; Hagan and Conner 2013; Malapi-Wight et al. 2014; Williams-Woodward 2014). Although fungicidal management of the pathogen is effective (Henricot et al. 2008; Henricot and Wedgwood 2013; LaMondia 2014), the use of genetic resistance is the preferred and likely most effective method for long-term plant disease management. Efforts have been made to screen boxwood genotypes for resistance using detached stem/branch assays in the laboratory (Ganci 2014; Guo et al. 2015; Shishkoff et al. 2015) or by using whole plants in the field (Ganci et al. 2013; Gehesquière 2014; Henricot et al. 2008). These resistance screening assays have all used fungal conidia as inoculum. However, the preparation of conidial inoculum is a time-consuming process, especially when there is a need to generate large amounts of inocula from many culture plates and when sporulation capacity varies across fungal isolates. Mycelium, if effective in causing symptoms, would be a much easier source of inoculum for these screening assays. In addition, a lab-based detached leaf screening assay is an efficient tool to rapidly evaluate large numbers of genotypes, and also

Corresponding author: Margaret Pooler; E-mail: margaret.pooler@ ars.usda.gov

Accepted for publication 1 March 2016.

http://dx.doi.org/10.1094/PDIS-01-16-0016-RE

This article is in the public domain and not copyrightable. It may be freely reprinted with customary crediting of the source. The American Phytopathological Society, 2016. would enable resistance assessment to be made under highly controlled conditions. However, a detached leaf assay is valuable only if it is highly correlated with the response of the whole plant. Our research focused on two objectives: i) to determine the parameters for using mycelium instead of conidia in a simple detached leaf assay, and ii) to validate the simple detached leaf assay by comparing it with symptom expression in whole boxwood plants.

\section{Materials and Methods}

Plant material. Five-year-old boxwood plants were provided by commercial growers (Saunders Brothers, Inc., Piney River, VA and Woodlanders, Inc., Aiken, SC). Eight cultivars were used in this study, including Buxus sempervirens 'Suffruticosa' as a highly susceptible control (Ganci et al. 2013) (Table 1). All plants were grown and maintained in high-tunnel polyhouses at the USDA-ARS station in Beltsville, MD. Houses were generally maintained at ambient outside temperatures except for in the winter, when they were kept above freezing. For whole-plant experiments, plants were moved into a temperature-controlled greenhouse 2 days prior to inoculation. For detached leaf experiments, terminal branches with two to six pairs of fully developed leaves were cut from whole boxwood plants described above, wrapped with moist paper towels, and stored in resealable plastic bags at $4{ }^{\circ} \mathrm{C}$ for up to 2 days prior to use. The second, third, or fourth pair of leaves (counted from the top) were used. Five plants of each cultivar and 10 leaves from each plant were used for the leaf assays.

Fungal isolates and growth. In order to avoid possible differential or interactive effects of fungal isolate and plant genotype, 10 C. pseudonaviculata isolates were used in this study. These isolates were provided by JoAnne Crouch (USDA-ARS, Systematic Mycology and Microbiology Laboratory, Beltsville, MD) and are classified as true C. pseudonaviculata (Gehesquière et al. 2016). They were originally isolated from Connecticut (CpsCT3, CpsCT9, CpsCT14, CpsCT15, CpsL1, and CpsS1), North Carolina (NCBB-1), or Oregon (ODA\#1) in the United States or from the United Kingdom (CBS114417) or New Zealand (ICMP17495). All cultures were grown and maintained on potato dextrose agar (PDA) plates at 8 to $10^{\circ} \mathrm{C}$ with subculturing every 3 months. Fresh cultures were obtained by transferring a single mycelial plug cut from the growing edge of a culture with a $10-\mathrm{mm}$ cork-borer onto the center of a new plate with an approximately 
$6 \mathrm{~mm}$ thick layer of PDA. Each seeded petri plate was sealed with Parafilm and incubated for 2 weeks at $23^{\circ} \mathrm{C}$ for inoculum preparation.

Inoculum preparation. Mycelium inoculum was prepared from the fungal culture plate. Agar disks were harvested from the growing edge of 2-week-old cultures using a 10-mm cork borer. Each myceliumcontaining agar disk was ground with a mortar and pestle with $1 \mathrm{ml}$ of sterile distilled water to homogenize mycelia. The resultant solution was diluted 10 times by adding $9 \mathrm{ml}$ sterile water to each homogenized disk for the detached leaf inoculation, and 20 times for the whole plant inoculation. Equal volumes of diluted solution from each of 10 isolates were combined and filtered through two to three layers of cheesecloth to remove large debris before use as mycelium inoculum. An agar disk with no mycelium was used as a no-inoculum control.

The spore inoculum was prepared from culture plates by following the procedure described by Guo et al. (2015). Briefly, the mycelium from the surface of the PDA plate was flattened and scraped with a sterile spatula. The scraped plates were placed into a $23^{\circ} \mathrm{C}$ incubator with $24 \mathrm{~h}$ constant light for 3 to 5 days. Conidia were collected by rinsing the culture surface with sterile water. The resultant spore solution was then filtered through two layers of cheesecloth to remove culture debris. Spore concentration was determined by hemacytometer counts under a microscope. A mixture with equal numbers of spores of each of the 10 isolates was diluted to the desired working concentration with sterile water and used as spore inoculum. Sterile distilled water without spores was used as a control.

Detached leaf inoculation. For each experiment, 10 leaves were used from each of five plants for a total of 50 detached leaves per cultivar. Terminal stems bearing two to six pairs of leaves were immersed in $70 \%$ ethanol for $5 \mathrm{~s}$, rinsed three times in sterile distilled water, and

Table 1. Boxwood (Buxus) taxa used to investigate Calonectria pseudonaviculata inoculation parameters and susceptibility to disease

\begin{tabular}{|c|c|}
\hline Accession $^{a}$ & Taxon \\
\hline NA 81312 & Buxus balearica Lam. \\
\hline NA 82631 & B. harlandii Hance \\
\hline NA 82656 & $\begin{array}{l}\text { B. microphylla Seibold \& Zucc.. 'Jim } \\
\text { Stauffer' }\end{array}$ \\
\hline NA 82632 & $\begin{array}{l}\text { B. microphylla Seibold \& Zucc.. 'John } \\
\text { Baldwin' }\end{array}$ \\
\hline NA 29703-H & B. sempervirens L. 'Suffruticosa' \\
\hline NA 6395-55 & B. sempervirens L. 'Vardar Valley' \\
\hline NA 82634 & $\begin{array}{l}\text { B. sinica var. insularis (Nakai) M. Cheng } \\
\text { 'Justin Brouwers' }\end{array}$ \\
\hline ‘NA 82635 & $\begin{array}{l}\text { B. sinica var. insularis (Nakai) M. Cheng } \\
\text { 'NaNa' }\end{array}$ \\
\hline
\end{tabular}

a NA is the National Arboretum accession number. Species authorities are based on taxonomy of the USDA-ARS Germplasm Resources Information Network (GRIN - www.ars-grin.gov) gently shaken to remove excess water. Leaves from the second to fourth pair were excised from stems by cutting at the base of the petiole.

Two inoculation methods were tested - pipetting liquid inoculum onto each leaf ("dropping"), or inserting each leaf into an actively growing fungal colony in agar ("plating"). For the dropping method (Fig. 1A), the petiole ends of excised leaves were inserted into $1.5 \%$ water agar in $100 \times 10 \mathrm{~mm}$ polystyrene petri dishes. Leaves were placed at an angle such that the abaxial (lower) side of the leaf faced upward. A 5-ul droplet of the mycelium inoculum (10× dilution) or conidia inoculation (500 spores) was pipetted onto the abaxial surface of each leaf, where it remained in place due to surface tension. Petri dishes with inoculated leaves were sealed with Parafilm and incubated at $23^{\circ} \mathrm{C}$ for 7 days (Guo et al. 2015). Infected area and total area of each leaf were measured using Assess 2.0, the Image Analysis Software for Plant Disease Quantification (APS Press, St. Paul, MN). Disease rating was expressed as the percentage of infected area over total area of each leaf.

For the plating method (Fig. 1B), excised leaves were inserted with 3 to $5 \mathrm{~mm}$ of the lateral edge of the leaf on the margins of a 2-week-old

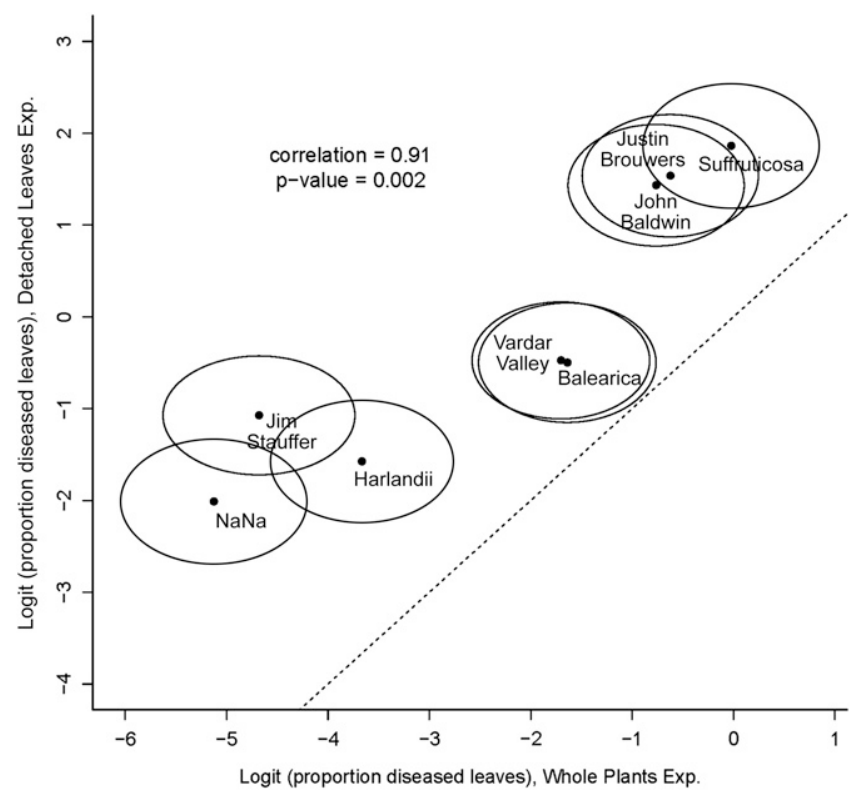

Fig. 2. Scatterplot of the logit of the means and their $95 \%$ confidence intervals (represented as ellipses) for whole plant assays and detached leaf assays for each cultivar. For reference, a logit of zero represents $50 \%$; logits increase as the percent increases and decrease (become more negative) as the percentage decreases toward zero.
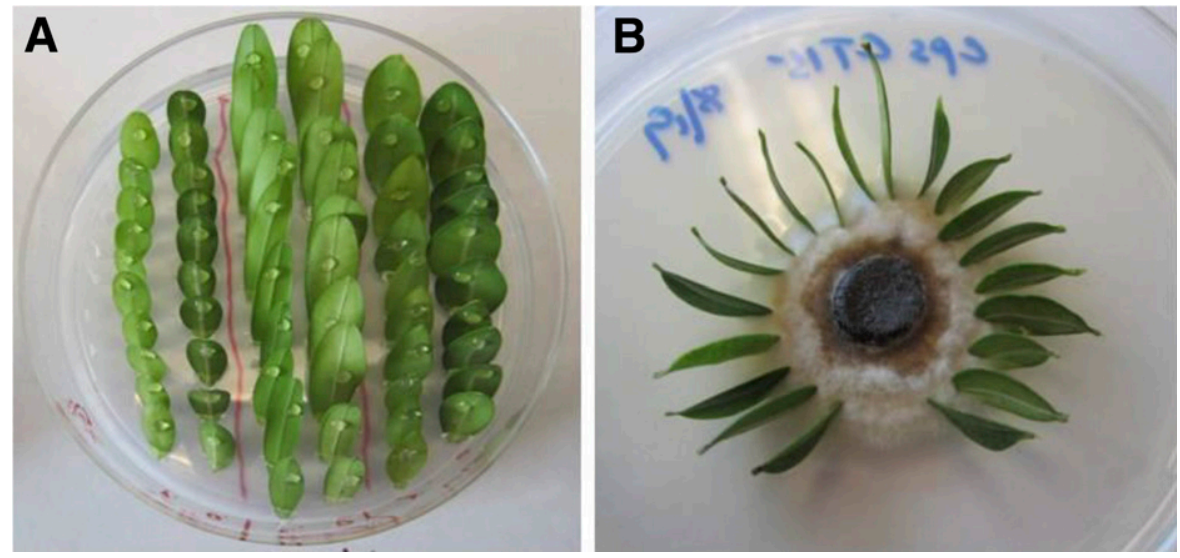

Fig. 1. Illustration of how detached leaves were inoculated using $\mathbf{A}$, the dropping method and $\mathbf{B}$, the plating method. For the dropping method, leaves were inserted into water agar and $5 \mu \mathrm{l}$ of inoculum was applied to the abaxial side. For the plating method, leaves were inserted on to the margins of a 2-week-old colony growing on agar plates. 
growing colony on agar plates. Ten leaves from each of five plants were placed around the colony margins on the plate containing the C. pseudonaviculata NCBB-1 isolate (isolated from NC). This isolate caused the most severe symptom expression among the isolates tested in previous laboratory assays (data not shown). Plates were sealed with Parafilm and incubated upside down at $23^{\circ} \mathrm{C}$. At the third day post inoculation, the leaves were transferred with a sterile forceps to a fresh $1.5 \%$ water agar plate by inserting the inoculated part of a leaf into agar. Plates were sealed with Parafilm and incubated at $23^{\circ} \mathrm{C}$ for 7 days, when data were collected as described above.

Whole plant inoculation. Five plants per cultivar were inoculated by spraying the inoculum (20x dilution) of mycelium or $2 \times$ $10^{4}$ conidia/ml on both adaxial and abaxial leaf surfaces with a mist sprayer by holding the plant upside down and spraying until runoff. Approximately 5 to $10 \mathrm{ml}$ inoculum was needed per plant depending on size. An additional five plants of the susceptible control, B. sempervirens 'Suffruticosa,' were sprayed with water as controls. Each inoculated plant was put into a 2-gallon sealed plastic bag and incubated in a greenhouse kept at 20 to $25^{\circ} \mathrm{C}$. The plastic bags with inoculated plants were kept completely zipped for 3 days to maintain high humidity, and then partially unzipped at the fourth day post inoculation. Disease ratings were taken on the seventh day post inoculation. Previous trials in our greenhouse indicated that symptom expression (as measured by total diseased leaves - both fallen and attached) did not increase after 7 days post inoculation, although more diseased leaves fell off over time. Diseased leaves were collected from each plant by gently shaking the plant, and all the leaves were counted and categorized - fallen, infected and attached, or noninfected and attached. The disease rating was expressed as the percentage of fallen or infected leaves over total leaves on the plant.

Whole plant and detached leaf inoculation experiments were conducted on three dates - late spring, late summer, and winter - to test the effect of plant physiological state on symptom expression.

Statistical methods. All experiments were conducted in a completely randomized design. For whole plant assays, the distribution of the proportion of fallen (i.e., diseased) leaves is binomial, and was analyzed in the generalized linear mixed models framework, with cultivar, inoculation method, and their interaction as fixed effects, and trial date (and its interactions with fixed effects) and plant as random effects variables. Trial date was considered a random

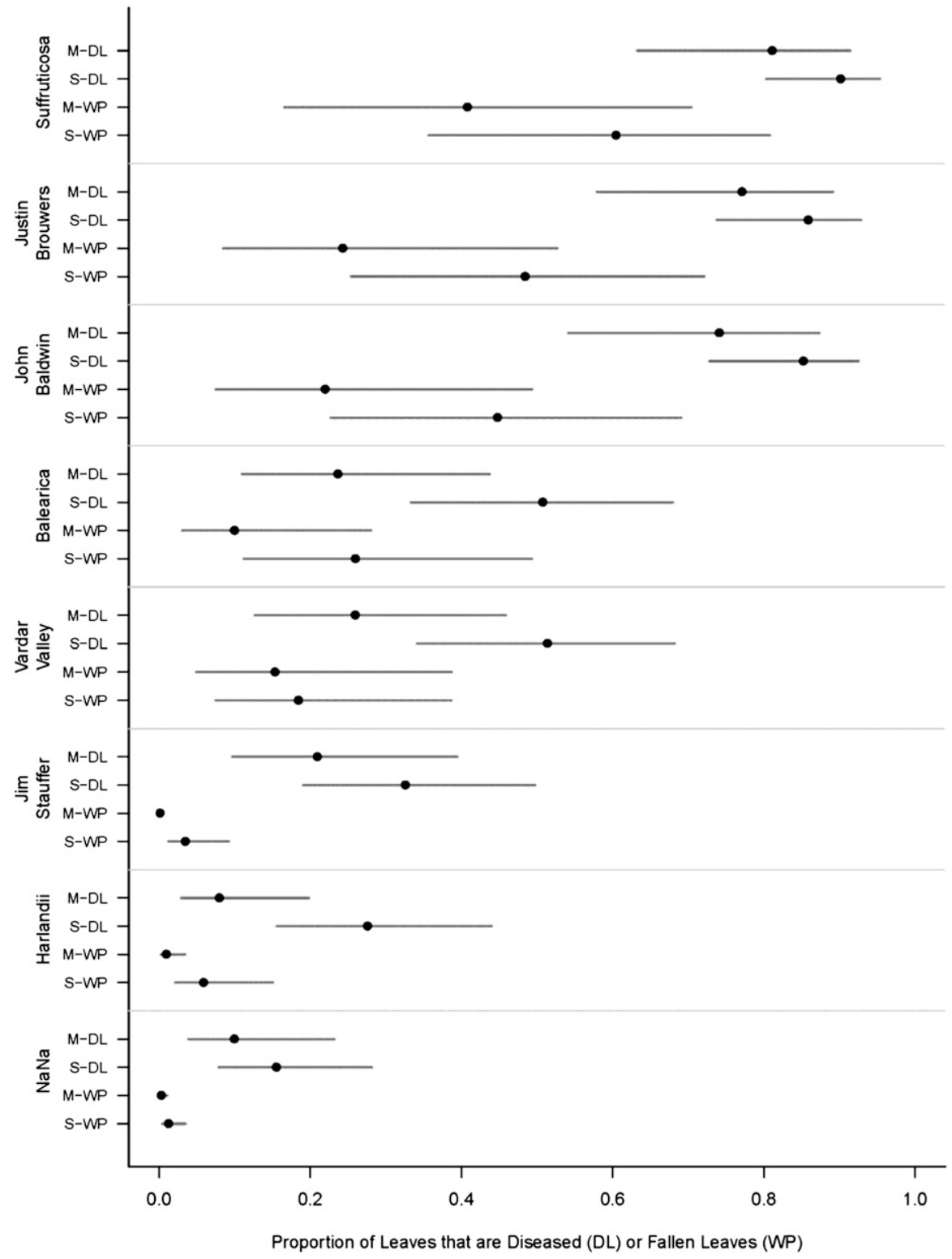

Fig. 3. Means and their $95 \%$ confidence intervals for disease ratings for whole plant assays (WP) and detached leaf assays (DL) for each cultivar, using spore (S) or mycelium (M) as inoculum. Means and their confidence intervals are back-transformed to the proportion scale for ease of interpretation. 
variable in the overall analysis to broaden the inference space for the effects of inoculation method. Models were estimated using the lme4 package (Bates et al. 2014) in R (R Core Team 2014). Variance decompositions were also calculated using this package, treating all variables as random. For detached-leaf assays, the distribution of proportion of diseased leaves for the whole data set was examined first. Of the 2,760 leaves, 1,188 had no diseased area, 987 had $100 \%$ diseased area, and the remaining 585 had an intermediate value. This produced a strong U-shaped (or bimodal) distribution, with most values either zero or one. Thus, the data were recoded as binomial, with each leaf with 5\% or more of its area diseased considered "diseased," otherwise "not diseased." The 5\% cutoff was used because the inoculation site accounted for up to $5 \%$ of the leaf area, and often caused the Assess software to classify the area as "diseased" even if there was no disease spread. Classifying the data as binomial made it possible to use the same statistical approach as was used for whole plant assays. A subset of the cultivars was modeled (those with less than $100 \%$ diseased leaves using the plating inoculation method) when testing the fixed effects of cultivar and inoculation method. When examining differences due to inoculation date, date was modeled as a fixed, rather than random, variable.

\section{Results}

Overall ranking of cultivars was well preserved between the wholeplant and the detached leaf assays (Fig. 2, correlation $=0.91, P=$ 0.002). Comparing the scales of the two axes in Figure 2, the whole plant assay was less sensitive than the detached leaf assay; that is, it had a consistently lower proportion of symptom expression. However, the relative rank of cultivars was similar for the two assays.

When the estimates were further partitioned by the type of inoculum used (spores versus mycelium), the overall ranking of cultivars was also preserved (Fig. 3). For whole plants, the interaction between cultivar and inoculum type was not significant (likelihood ratio [LR] test, $\chi^{2}=8.55$, df $=7, P=0.29$ ), supported by a variance decomposition which found the main effects of cultivar $(90.0 \%$ of the variance) and inoculum type $(1.4 \%)$ to be the only important fixed effects. The contributing random effects were trial date $(0.6 \%)$ and cultivar by trial date interaction $(8.9 \%)$. Thus, the small but significant (LR test, $\chi^{2}=4.80, \mathrm{df}=1, P=0.03$ ) difference between inoculum types was consistent among cultivars (Fig. 3 ).

For detached leaves, neither the inoculum type nor its interaction with cultivar were significant (LR tests, $\chi^{2}=5.57$, df $=7, P=0.59$; $\chi^{2}=1.98, \mathrm{df}=1, P=0.16$, respectively), again consistent with a variance decomposition where only cultivar $(91.0 \%)$ and trial date $(8.8 \%)$ were important. Thus, both inoculum types produced the same results for all cultivars (Fig. 3), indicating that mycelium can be used as inoculum in assays using either detached leaves or whole plants.

Detached leaves were also used to determine if the two leaf inoculation methods, dropping and plating, both using a mycelium inoculum, differed. The proportion of diseased leaves was much higher for plating (results not shown), so much so that for three of the relatively susceptible accessions (B. balearica, 'Justin Brouwers,' and 'Suffruticosa'), $100 \%$ of leaves were categorized as diseased. Thus, the plating inoculation method would not be suitable for discriminating among susceptible cultivars. Fitting mixed models to the remaining five more resistant cultivars, indicated both a significant interaction between cultivar and inoculation method (LR test, $\chi^{2}=20.82, \mathrm{df}=4, P<$ 0.001 ), and a significant main effect of inoculation method (LR test, $\left.\chi^{2}=8.15, \mathrm{df}=1, P=0.004\right)$. Thus, the difference between the two leaf inoculation methods was not consistent among cultivars, although plating always produced more diseased leaves. For these five more resistant cultivars, plating provided better discrimination between them; the variance of the cultivar model coefficients for plating, on the logit scale, was 2.7 , versus 1.0 for dropping.

To investigate the effect of season or plant physiological state on inoculating detached leaves, inoculation date was treated as a fixed effect, and we asked whether the pattern of cultivar resistance differed among the dates (Fig. 4). While the ranking stayed much the same over dates, clustering of the cultivars changed. In the 15 Jan spore trial, the cultivars were well spread across the proportion of diseased leaves. In all other trials, the three susceptible varieties clustered together and were separated from the five more resistant varieties. The variance of the means of the eight cultivars for each date-type combination was calculated and used as a proxy for the amount of separation among the cultivars. This model resulted in the ranked variances as follows: 3.6 (23 Sept-spores), 3.4 (15 Jan-spores), 3.0 (23 Sept-mycelium), 2.2 (26 June-mycelium), and 1.2 (30 Apr-spores). Thus, there appears to be slightly better separation using spores than mycelium (comparing the two only for the 23 Sept date), and neither the April nor the June date separated the cultivars as well as the others. The interaction of date-method by cultivar and the main effect of date-method are significant (LR tests, $\chi^{2}=47.0, \mathrm{df}=28, P=$ $0.014 ; \chi^{2}=151.6$, df $=4, P<0.001$, respectively). Figure 4 also shows that the inoculation date had its largest effect on cultivars of intermediate resistance. These cultivars tended to cluster with the more resistant genotypes on the 23 Sept date, but less so on the other dates. Overall, however, assays performed in the spring, summer, or winter gave comparable results in terms of identifying the most resistant genotypes.

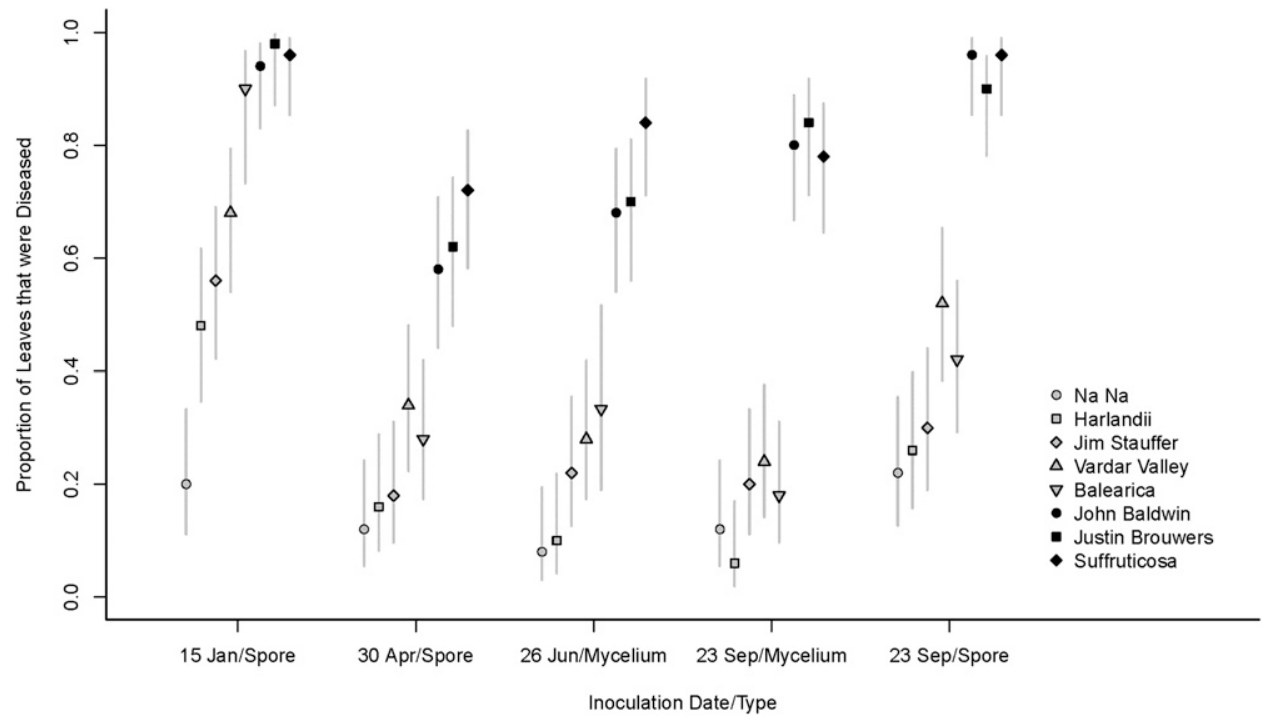

Fig. 4. Means and their $95 \%$ confidence intervals for disease ratings for detached leaf assays for each cultivar and each inoculation date/inoculation type (spore or mycelium) combination. Means and their confidence intervals are back-transformed to the proportion scale for ease of interpretation. Cultivars are ordered by overall average proportion, with 'NaNa' the most resistant and 'Suffruticosa' the least resistant. 


\section{Discussion}

The primary purpose of these experiments was to develop a rapid and effective screening assay for boxwood blight resistance. An ideal assay would allow screening of hundreds of accessions and potentially thousands of seedlings, could be done in the laboratory where the pathogen could be isolated from landscapes where it was not yet present, and would also utilize detached leaves or branches instead of whole plants so that numerous accessions could be tested in a relatively small amount of space. Finally, the inoculation material and method would be relatively simple and easy to prepare.

Our experiments that directly compared whole-plant inoculation to detached leaves indicate that laboratory screening using leaves was comparable to inoculation and disease rating of whole plants in terms of identifying the most resistant genotypes (Fig. 2). The disease incidence was less with whole plants (measured by percentage of leaf drop) compared with detached leaves (measured by percentage of leaves expressing symptoms), but both methods consistently identified the most susceptible and resistant genotypes.

There have been no systematic studies to determine when boxwood plants are most susceptible to blight, or whether resistance screening assays can be performed at any time during the year. This study therefore tested plants in different physiological states during different seasons, including in the spring during active growth, in the late summer, and in the winter when plants were dormant. Although disease symptom expression was higher on actively growing plants, the relative disease resistance was consistent among cultivars regardless of time of inoculation and physiological state of the plant. Screening assays can therefore be performed at essentially any time of the year in the laboratory with accurate results.

The vast majority of studies of plant resistance to fungal pathogens, including all those dealing with boxwood blight, have utilized fungal spores (conidia) as the inoculum. Mycelia, while faster to grow and scale to larger quantities, have been used as inoculum in only a few reports (e.g., Chen and Wang 2005; Park et al. 2008; Singh et al. 2002). Accurate quantitation of mycelium can be an issue, as standard measurement methods such as $\mathrm{OD}_{600}$ or $\mathrm{CFU}$ are not as accurate for mycelium as spores. However, mycelium quantities can be optimized and standardized within an experiment by dilutions of consistent explant material from the agar plate. Our study is the first to systematically compare the two types of inoculum in C. pseudonaviculata. Symptom expression tended to be slightly more pronounced in both the whole plant and the detached leaf assays when spores were used compared with mycelium (Fig. 3). However, the relative rankings of cultivars, and equally important, the ability to separate most resistant from least resistant cultivars, was similar for both inoculum types.

The methods reported here provide an efficient and reliable assay to screen hundreds of existing boxwood cultivars and germplasm accessions and potentially thousands of hybrid seedlings for resistance to boxwood blight. This assay enabled consistent differentiation between the most tolerant and most susceptible boxwood genotypes. While most boxwood species appear to be at least somewhat susceptible to the pathogen (Henricot et al. 2008), identification of the least susceptible genotypes will be useful for informing best management practices in new boxwood plantings and for developing new blighttolerant cultivars.

\section{Literature Cited}

Bates, D., Maechler, M., Bolker, B., and Walker, S. 2014. lme4: Linear mixedeffects models using Eigen and S4. R package version 1.0-6. Online: http://CRAN. R-project.org/package=lme4

Chen, Y., and Wang, D. 2005. Two convenient methods to evaluate soybean for resistance to Sclerotinia sclerotiorum. Plant Dis. 89:1268-1272.

Elmhirst, J. F., and Auxier, B. E. 2013. First report of box blight caused by Cylindrocladium pseudonaviculata $(C$. buxicola) in British Columbia, Canada. Plant Dis. 97:559.

Ganci, M. L. 2014. Investigation of host resistance in Buxus species to the fungal plant pathogen Calonectria pseudonaviculata $(=$ Cylindrocladium buxicola), the causal agent of boxwood blight and determination of overwinter pathogen survival. M.S. thesis, North Carolina State University, Raleigh, NC.

Ganci, M., Benson, D. M., and Ivors, K. L. 2013. Susceptibility of commercial boxwood varieties to Cylindrocladium buxicola. NCSU Cooperative Extension. Online: http://www.ces.ncsu.edu/wp-content/uploads/2013/05/final-2012-cult-suscsummary.pdf

Gehesquière, B. 2014. Cylindrocladium buxicola nom. cons. prop. (syn. Calonectria pseudonaviculata) on Buxus: molecular characterization, epidemiology, host resistance and fungicide control. Ph.D. thesis, Ghent University, Belgium.

Gehesquière, B., Crouch, J. A., Marra, R. E., VanPoucke, K., Rys, F., Maes, M., Gobin, B., Hofte, M., and Heungens, K. 2016. Characterization and taxonomic re-assessment of the box blight pathogen Calonectria pseudonaviculata, introducing Calonectria henricotiae sp. nov. Plant Pathol. 65:37-52.

Guo, Y. H., Olsen, R. T., Kramer, M., and Pooler, M. 2015. Effective bioassays for evaluating boxwood blight susceptibility using detached stem inoculations. HortScience 50:268-271.

Hagan, A. K., and Conner, K. 2013. Boxwood blight - a new disease of boxwood in the nursery and landscape in Alabama. Alabama Cooperative Extension System, Auburn, AL. Online: http://alfafarmers.org/uploads/files/Boxwood_Blight_ACES.pdf

Henricot, B., and Culham, A. 2002. Cylindrocladium buxicola, a new species affecting Buxus spp., and its phylogenetic status. Mycologia 94:980-997.

Henricot, B., Gorton, C., Denton, G., and Denton, J. 2008. Studies on the control of Cylindrocladium buxicola using fungicides and host resistance. Plant Dis. 92: 1273-1279.

Henricot, B., Perez Sierra, A., and Prior, C. 2000. A new blight disease on Buxus in the UK caused by the fungus Cylindrocladium. Plant Pathol. 49:805.

Henricot, B., and Wedgwood, E. 2013. Evaluation of foliar fungicide sprays for the control of boxwood blight, caused by the fungus Cylindrocladium buxicola. Online. Plant Health Prog. doi:10.1094/PHP-2013-1024-01-RS.

Ivors, K. L., Lacey, L. W., Milks, D. C., Douglas, S. M., Inman, M. K., Marra, R. E., and LaMondia, J. A. 2012. First report of boxwood blight caused by Cylindrocladium pseudonaviculata in the United States. Plant Dis. 96:1070.

LaMondia, J. A. 2014. Fungicide efficacy against Calonectria pseudonaviculata, causal agent of boxwood blight. Plant Dis. 98:99-102.

Malapi-Wight, M., Hebert, J. B., Buckley, R., Daughtrey, M. L., Gregory, N. F., Rane, K., Tirpak, S., and Crouch, J. 2014. First report of boxwood blight caused by Calonectria pseudonaviculata in Delaware, Maryland, New Jersey and New York. Plant Dis. 98:698.

Park, D.-S., Sayler, R. J., Hong, Y.-G., Nam, M.-H., and Yang, Y. 2008. A method for inoculation and evaluation of rice sheath blight disease. Plant Dis. 92:25-29.

R Core Team. 2014. R: A language and environment for statistical computing. R Foundation for Statistical Computing, Vienna, Austria. Online: http://www. R-project.org

Shishkoff, N., Daughtrey, M., Aker, S., and Olsen, R. T. 2015. Evaluating boxwood susceptibility to Calonectria pseudonaviculata using cuttings from the National Boxwood Collection. Plant Health Prog. 16:11-15.

Singh, A., Rohilla, R., Singh, U. S., Savary, S., Willocquest, L., and Duveiller, E. 2002. An improved inoculation technique for sheath blight of rice caused by Rhizoctonia solani. Can. J. Plant Pathol. 24:65-68.

USDA-NASS. 2010. Census of Horticultural Specialties (2009). Online: http:// www.agcensus.usda.gov/Publications/2007/Online_Highlights/Census_of_ Horticulture_Specialties/

Williams-Woodward, J. L. 2014. Boxwood blight found in Georgia - disease alert. University of Georgia Extension, Athens, GA. Online: http://plantpath.caes uga.edu/extension/extension/documents/GABoxwoodBlightAlertv1.pdf 